\title{
A simple approach to solve boundary-value problems in gradient elasticity
}

\author{
C. Q. Ru and E. C. Aifantis, Houghton, Michigan
}

(Received May 19, 1992)

Summary. We outline a procedure for obtaining solutions of certain boundary value problems of a recently proposed theory of gradient elasticity in terms of solutions of classical elasticity. The method is applied to illustrate, among other things, how the gradient theory can remove the strain singularity from some typical examples of the classical theory.

\section{Introduction}

A higher-order strain gradient theory has been proposed by Aifantis and co-workers [1]- [4], in order to address the heterogeneity and pattern development in elastic deformation and plastic flow. In the case of plasticity, the gradient theory predicts finite thicknesses for shear bands, as well as spacings and velocities for travelling deformation bands. In examining the effect of gradients on the structure of the crack tip, in particular, Altan and Aifantis [5] adopted a simple constitutive equation of the form

$\sigma=\lambda(\operatorname{tr} \varepsilon) I+2 \mu \varepsilon-c \nabla^{2}[\lambda(\operatorname{tr} \varepsilon) I+2 \mu \varepsilon]$,

where $(\sigma, \varepsilon)$ are the stress and strain, $(\lambda, \mu)$ the Lamé constants, $\nabla^{2}$ the Laplacian and $c$ a constant gradient coefficient. Making use of (1), Altan and Aifantis have solved the mode-III crack problem and found that the strain is finite at the crack tip but the stress remains singular, as in the classical theory. In this paper we report additional interesting implications of (1) for some typical problems which are locally singular within the framework of classical elasticity theory. In doing so, a few general results are obtained which allow a direct relation between solutions to boundary value problems of (1) and solutions corresponding to the classical theory $(c \equiv 0)$.

On introducing (1) with $c \equiv 0$ and $c \neq 0$ into the equilibrium equations (div $\sigma=0$ ) we obtain the following differential equations for the displacement vector $\boldsymbol{u}\left(2 \boldsymbol{\varepsilon}=\nabla \boldsymbol{u}+[\boldsymbol{\nabla u}]^{T}\right)$ :

$\boldsymbol{L} \boldsymbol{u}^{0}=0, \quad \boldsymbol{L} \equiv \mu \nabla^{2}+(\lambda+\mu) \operatorname{grad} \operatorname{div}$,

for the classical case $(c \equiv 0)$, and

$\left(1-c \nabla^{2}\right) L u=0$

for the gradient-dependent case $(c \neq 0)$. In general, one has to solve the fourth-order partial differential equation (3) subject to appropriate boundary conditions. Nevertheless, the following observation can reduce the complexity of this task and greatly facilitate the obtaining of solutions in certain cases. 
Due to the fact that the operators $L$ and $\nabla^{2}$ commute, it is noted from (3) that the vector field $\left(1-c \nabla^{2}\right) \boldsymbol{u}$ satisfies the classical equilibrium equations (2). Thus, if $\left(1-c \nabla^{2}\right) \boldsymbol{u}$ could be identified with the classical displacement field $\boldsymbol{u}^{0}$ of a certain classical boundary-value problem of linear elasticity which can readily be solved, then the original fourth-order problem (3) is reduced into the following second-order problem:

$\left(1-c \nabla^{2}\right) \boldsymbol{u}=\boldsymbol{u}^{0}$.

Obviously, the solution of (4) is more conveniently obtained and, in doing so, a connection between the "gradient" and the "classical" solutions is readily established. Moreover, it turns out that (4) is further simplified when it is used to obtain the structure of the displacement (and stress) field near singularities (and discontinuities). The details of the above ideas and some typical examples will be explained in the following Sections.

\section{General results}

First, we establish the following straight-forward result for the case of traction boundary value problems.

Theorem 1: Let the traction vector $s$ be prescribed at the whole boundary $S$ (enclosing the region $V$ )

$\boldsymbol{s}=\boldsymbol{\sigma n}=\boldsymbol{s}_{0} \quad$ on $S$,

where $\boldsymbol{n}$ is the unit vector normal to $S$ and $\boldsymbol{\sigma}$ is given by (1). Then the gradient solution $\boldsymbol{u}$ of (3) satisfies (4) where $\boldsymbol{u}^{0}$ is the classical solution of (2) satisfying the same traction boundary condition.

In other words, $\boldsymbol{u}^{0}$ is obtained from the classical boundary value problem

$u^{0}: L u^{0}=0 \quad$ in $V ; \quad \sigma^{0} n=s_{0} \quad$ on $S$,

where $\sigma^{0}$ is expressed in terms of $\boldsymbol{u}^{0}$ through the classical Hooke's law

$\boldsymbol{\sigma}^{0}=\boldsymbol{H} \boldsymbol{\varepsilon}^{0} \equiv \lambda\left(\operatorname{tr} \boldsymbol{\varepsilon}^{0}\right) I+2 \mu \varepsilon^{0}$,

$\boldsymbol{\varepsilon}^{0}=D \boldsymbol{u}^{0} \equiv \frac{1}{2}\left(\nabla \boldsymbol{u}^{0}+\left(\nabla \boldsymbol{u}^{0}\right)^{T}\right)$,

with $T$ denoting, as usual, transposition.

Proof: It follows from (3) and (1) that $\left(1-c V^{2}\right) \boldsymbol{u}$ satisfies the classical equilibrium equation (2) and also the classical constitutive equations (7), i.e.

$\sigma=H D\left(1-c \nabla^{2}\right) \boldsymbol{u}$.

In view of (5), (6.2) and the uniqueness of $\boldsymbol{u}^{0}$, it then follows that $\left(1-c V^{2}\right) \boldsymbol{u}$ satisfies (4) and that

$\sigma=\sigma^{0}$.

This result, i.e. Eq. (10), has also been obtained by Altan and Aifantis [5] for the mode-III crack problem. [In this connection, it is emphasized that the validity of conclusions (4) and (10) of Theorem 1 has nothing to do with the extra gradient boundary conditions required as a result of the higher gradient terms in (3)]. 
Next, in line with Altan and Aifantis [5], we adopt the following extra boundary condition: $\frac{\partial^{2} \boldsymbol{u}}{\partial n^{2}}=0 \quad$ on $S$.

Then, it turns out that $\boldsymbol{u}$ can equivalently be found as the solution of the problem

$\boldsymbol{u}:\left(1-c \nabla^{2}\right) \boldsymbol{u}=\boldsymbol{u}^{0} \quad$ in $V, \quad \frac{\partial^{2} \boldsymbol{u}}{\partial n^{2}}=0 \quad$ on $S$.

It remains to consider existence and uniqueness of the solution but this general question is not addressed here. Nevertheless, the examples of Section 3 indicate that the problem (12) is conveniently solved for many important cases and that uniqueness is ensured.

For displacement boundary conditions, the conclusions of Theorem 1 are no longer valid, in general. However, the following result holds for a large class of problems.

Theorem 2: Let the traction $s$ be prescribed in a part $S_{1}$ of $S$, and the displacement $u$ be prescribed in the remainder part $S_{2}$, where $S=S_{1}+S_{2}$ and $S_{2}$ is located at a plane (say, $y \equiv 0$ ), i.e.,

$L\left(1-c \nabla^{2}\right) \boldsymbol{u}=0 \quad$ in $V$,

$\boldsymbol{\sigma n}=s_{0} \quad$ on $S_{1}$,

$\frac{\partial^{2} \boldsymbol{u}}{\partial n^{2}}=0 \quad$ on $S_{1}$

$\boldsymbol{u}=\boldsymbol{u}_{0} \quad$ on $\mathrm{S}_{2}$

$\frac{\partial^{2} \boldsymbol{u}}{\partial n^{2}} \equiv \frac{\partial^{2} \boldsymbol{u}}{\partial y^{2}}=0 \quad$ on $S_{2}$.

Then relationship (4) is still true provided that $\boldsymbol{u}^{0}$ is the solution of the following problem:

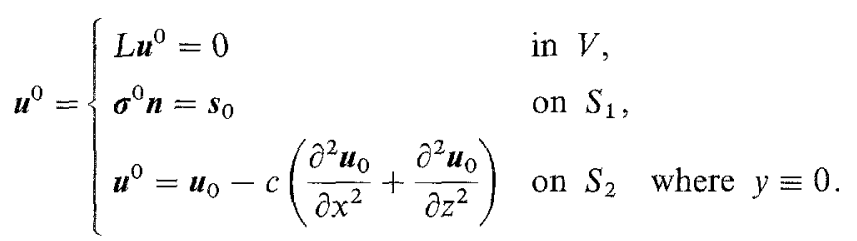

Proof: To establish the proof, it is sufficient to verify that $\left(1-c \nabla^{2}\right) \boldsymbol{u}$ satisfies indeed the displacement boundary condition (14.3) on $S_{2}$.

In fact, from (13.5), we have

$\nabla^{2} \boldsymbol{u}=\frac{\partial^{2} \boldsymbol{u}}{\partial x^{2}}+\frac{\partial^{2} \boldsymbol{u}}{\partial z^{2}} \quad$ on $S_{2}$

It then follows from (13.4) that

$\nabla^{2} \boldsymbol{u}=\frac{\partial^{2} \boldsymbol{u}_{0}}{\partial x^{2}}+\frac{\partial^{2} \boldsymbol{u}_{0}}{\partial z^{2}} \quad$ on $S_{2}$,

or that $\left(1-c \nabla^{2}\right) u$ satisfies $(14.3)$.

Obviously, for the cases discussed in Theorem 2, we still have the result of (10). Therefore, to obtain the stress field $\sigma$, it is sufficient to know the classical stress field $\sigma^{0}$ corresponding to $\boldsymbol{u}^{0}$ defined by (14). This reduces the gradient elasticity problem (13) into a classical problem (14). 
Finally, it may be readily seen that Theorem 2 may be extended to more general cases where $S_{2}$ is composed of a finite number of planes, and further, by combining the Theorems 1 and 2, conclusions (4) and (10) may be extended to the cases of mixed displacement-traction boundary-valued problems.

\section{Examples}

\subsection{Crack problems}

Consider a homogeneous material in the $(x, y)$ plane, in which a crack is located at $-a \leqq x \leqq+a, y=0$.

Assume that tractions are prescribed on the crack surfaces, and let $\boldsymbol{u}$ and $\boldsymbol{u}^{0}$ be the "gradient" and "classical" solutions, respectively (corresponding to the same traction boundary value). Adopt the extra boundary condition (11), and let

$\left(\boldsymbol{u}_{+}{ }^{0}-\boldsymbol{u}_{-}{ }^{0}\right)=\boldsymbol{g}^{0}(x), \quad-a \leqq x \leqq a, \quad y=0$.

$\left(\boldsymbol{u}_{+}-\boldsymbol{u}_{-}\right)=g(x), \quad-a \leqq x \leqq a, \quad y=0$

be the crack-opening displacements. It then follows from (12) that $\left({ }^{\prime} \equiv d / d x\right)$

$g-c g^{\prime \prime}=g^{0}$.

This gives a very simple relationship between both the "gradient" and "classical" crack-opening displacements. The general solution of (15) for $c>0$ is

$g(x)=\frac{-1}{\sqrt{c}} \int_{x_{0}}^{x} \operatorname{sh}\left(\frac{x-t}{\sqrt{c}}\right) g^{0}(t) d t+c_{1} e^{x / \sqrt{c}}+c_{2} e^{(-x) / \sqrt{c}}, \quad-a \leqq x \leqq a$,

where $x_{0}$ may be chosen arbitrarily and $c_{1}, c_{2}$ are arbitrary constant vectors.

For example, for the classical constant traction crack problems, we have that

$g^{0}(x)=b \sqrt{a^{2}-x^{2}}, \quad-a \leqq x \leqq a$,

where the constant vector $\boldsymbol{b}$ depends on the applied traction and the elastic constants (e.g. for mode-III, $b=\frac{\tau_{0}}{\mu}$, where $\tau_{0}$ is the boundary traction).

If one requires that

$g(-a)=g(a)=0$,

then $g(x)$ is found from (16) as

$g(x)=\frac{\operatorname{sh}\left(\frac{a+x}{\sqrt{c}}\right)}{\sqrt{c} \operatorname{sh}\left(\frac{2 a}{\sqrt{c}}\right)} \int_{-a}^{+a} \operatorname{sh}\left(\frac{a-t}{\sqrt{c}}\right) g^{0}(t) d t-\frac{1}{\sqrt{c}} \int_{-a}^{x} \operatorname{sh}\left(\frac{x-t}{\sqrt{c}}\right) g^{0}(t) d t$. 
Substitution of (17) into (19) gives the gradient crack opening displacement as

$g(x)=\frac{b \operatorname{sh}\left(\frac{a+x}{\sqrt{c}}\right)}{\sqrt{c} \operatorname{sh}\left(\frac{2 a}{\sqrt{c}}\right)} \int_{-a}^{+a} \operatorname{sh}\left(\frac{a-t}{\sqrt{c}}\right) \sqrt{a^{2}-t^{2}} d t-\frac{b}{\sqrt{c}} \int_{-a}^{x} \operatorname{sh}\left(\frac{x-t}{\sqrt{c}}\right) \sqrt{a^{2}-t^{2}} d t$.

In particular, in contrast to the classical solution $g^{0}(x)$, the gradient solution $g(x)$ has a finite first derivative at the crack-tip, i.e.,

$$
g^{\prime}(-a)=\frac{b \int_{-a}^{a} \operatorname{sh}\left(\frac{a-t}{\sqrt{c}}\right) \sqrt{a^{2}-t^{2}} d t}{c \operatorname{sh}\left(\frac{2 a}{\sqrt{c}}\right)} \neq 0 .
$$

In addition, from (16) and (17), we see that $g^{\prime \prime}(-a)=g^{\prime \prime}(a)=0$.

In [5], Altan and Aifantis have adopted condition (11) for the whole axis of $x$ and therefore, from (5), one arrives at

$\boldsymbol{u}(x, 0)-c \boldsymbol{u}^{\prime \prime}(x, 0)=\boldsymbol{u}^{0}(x, 0), \quad 0<x<\infty$,

where $\boldsymbol{u}^{0}$ is the classical displacement. In particular, for $x \geqq a, \boldsymbol{u}^{0}(x, 0) \equiv 0$ and thus $\boldsymbol{u}(x, 0)$ has the form $c_{1} e^{-(x t / v)}$, for $x \geqq a$, where $c_{1}$ is a constant. This is in agreement with the result of [5] where $c_{1}=\frac{\pi \tau_{0} a}{2 \mu} I_{1}\left(\frac{a}{\sqrt{c}}\right)$ and $I_{1}$ is the modified Bessel function.

It is pointed out that if we assume that $c<0$, the general solution of (15) is

$g(x)=\frac{1}{\sqrt{-c}} \int_{-a}^{x} \sin \frac{x-t}{\sqrt{-c}} g^{0}(t) d t+c_{1} \sin \frac{x}{\sqrt{-c}}+c_{2} \cos \frac{x}{\sqrt{-c}}$

Furthermore, from (18), we have the following crack opening displacement:

$g(x)=\frac{1}{\sqrt{-c}} \int_{-a}^{x} \sin \frac{x-t}{\sqrt{-c}} g^{0}(t) d t-\frac{1}{\sqrt{-c}} \frac{\sin \frac{x+a}{\sqrt{-c}}}{\sin \frac{2 a}{\sqrt{-c}}} \int_{-a}^{a} \sin \frac{a-t}{\sqrt{-c}} g^{0}(t) d t$

and its first derivative at the crack-tip reads

$g^{\prime}(-a)=\frac{1}{c \sin \frac{2 a}{\sqrt{-c}}} \int_{-a}^{a} \sin \frac{a-t}{\sqrt{-c}} g^{0}(t) d t$

suggesting that the crack surfaces may have an oscillatory or undulated profile (but not interpenetrated if $c$ is sufficiently small). It turns out that if $(2 a / \sqrt{-c})=n \pi$ where $n$ is an integer, then the crack faces (profile) may pick more than one wavelength (non-uniqueness). 


\subsection{Flamant problem}

Consider the so-called Flamant problem for the half-plane $y \leqq 0$, with a (vertical) concentrated force $P$ at the origin. The classical displacement fields are given in [6] as

$u_{x}^{o}=\frac{P}{2 \pi(\lambda+\mu)}\left(\theta-\frac{\pi}{2}\right)+\frac{P x y}{2 \pi \mu r^{2}}$,

$u_{y}{ }^{0}=\frac{P(\lambda+2 \mu)}{2 \pi \mu(\lambda+\mu)} \log r-\frac{P x^{2}}{2 \pi \mu r^{2}}$,

where $u_{y}{ }^{0}$ has a singularity at the origin and $r^{2}=x^{2}+y^{2}$. From Theorem 1, the gradient field $\boldsymbol{u}$ may be found from (12). Let the surface displacements be $u_{y}(x, 0)=h(r), u_{y}{ }^{0}(x, 0)=h^{0}(r)$, at $y \equiv 0$. Then we have $\left({ }^{\prime} \equiv d / d r\right)$

$h-c h^{\prime \prime}=h^{0}=\frac{P(\lambda+2 \mu)}{2 \pi \mu(\lambda+\mu)} \log r-\frac{P}{2 \pi \mu}$,

whose solution reads

$h=\frac{-P(\lambda+2 \mu)}{4 \pi \mu \sqrt{c}(\lambda+\mu)} \int_{0}^{r}\left(e^{(r-t) / V \bar{c}}-e^{(t-r) / / \bar{c}}\right) \log t d t+c_{1} e^{r / \bar{c}}+c_{2} e^{(-r) / V \bar{c}}-\frac{P}{2 \pi \mu}$,

where $c_{1}$ and $c_{2}$ are undetermined constants.

It turns out that $c_{1}$ should be chosen such that to eliminate the exponential singularity $e^{r / d \bar{c}}$ as $r \rightarrow+\infty$, i.e.

$c_{1}=\frac{P(\lambda+2 \mu)}{4 \pi \mu \sqrt{c}(\lambda+\mu)} \int_{0}^{\infty} e^{(-t) / \bar{c}} \log t d t$.

Furthermore, one may note that $h^{\prime}(0)=\frac{1}{\sqrt{c}}\left(c_{1}-c_{2}\right)$ is always finite. It is thus natural to assume that $u_{y}(x, 0)$ has a continuous first derivative at $x=0$ and therefore $h^{\prime}(0)=0$. Thus, it may be concluded that $c_{1}=c_{2}$ and that

$h=\frac{P(\lambda+2 \mu)}{2 \pi \mu \sqrt{c}(\lambda+\mu)}\left[e^{(-r) / 2 \bar{c}} \int_{0}^{r} \operatorname{ch} \frac{t}{\sqrt{c}} \log t d t+\operatorname{ch} \frac{r}{\sqrt{c}} \int_{r}^{\infty} e^{(-t) / \sqrt{c}} \log t d t\right]$,

where an unimportant constant $\left(-\frac{P}{2 \pi \mu}\right)$ has been omitted.

Obviously, the singularity at $x=0$ disappears in the expression (24) and this is another classical case for which the gradient term eliminates a physically undesirable singularity.

For the 3-dimensional axisymmetric (the so-called Boussinesq) problem, a similar procedure leads to the following Bessel's equation:

$h-c\left(h^{\prime \prime}+\frac{1}{r} h^{\prime}\right)=\frac{b}{r}$,

where $b$ is a constant. The discussion of this problem is postponed until a future more detailed paper [8]. 


\subsection{Punch problem on half-plane}

As a simple application of Theorem 2, we consider the following punch problem for the half-plane $y \leqq 0$ :

$$
\begin{array}{ll}
\sigma_{y y}=\sigma_{x y}=0 & \text { at } y=0, \quad x<-a \text { or } x>a, \\
\boldsymbol{u}=\boldsymbol{u}_{0} & \text { at } y=0, \quad-a \leqq x \leqq a .
\end{array}
$$

From Theorem 2, let $\boldsymbol{u}^{0}$ be the classical solution of the following boundary value problem:

$\sigma_{y y}^{0}=\sigma_{x y}^{0}=0 \quad$ at $\quad y=0, \quad x<-a$ or $x>a$,

$\boldsymbol{u}^{0}=\boldsymbol{u}_{0}-c \boldsymbol{u}_{0}{ }^{\prime \prime}$ at $y=0, \quad-a \leqq x \leqq a$.

Then, (5) and (11) will hold. Thus, the punch problem of gradient elasticity may be reduced to another punch problem of classical elasticity.

In particular, if $\boldsymbol{u}_{0}{ }^{\prime \prime}=0, \boldsymbol{u}^{0}$ and $\boldsymbol{u}$ have the same boundary value. In other words, the gradient solution and the classical solution give the same stress distribution. However, it should be noticed that the discontinuity of $\boldsymbol{u}_{0}{ }^{\prime}$ will give rise to a singular boundary value of $\boldsymbol{u}^{0}$.

\section{Interface problems}

In this Section we consider a certain class of interface problems where two different gradient-dependent elastic materials are bonded by a plane (say, $y=0$ ) and their gradient coefficients are the same $\left(c_{1}=c_{2}=c\right)$. In this case, we can establish the following

Theorem 3: Suppose that

(i) the traction $s$ is prescribed at the whole boundary $S$,

(ii) $c_{1}=c_{2}=c$,

(iii) the following interfacial conditions hold ${ }^{1}$ :

$[\boldsymbol{u}]_{-}^{+}=[\boldsymbol{\sigma n}]_{-}^{+}=\left[\frac{\partial^{2} \boldsymbol{u}}{\partial n^{2}}\right]_{-}^{+}=0$

where []$_{-}{ }^{+}$denotes the difference of the respective quantity for the two materials. Then relationships (4) and (10) still hold throughout the whole domain occupied by the two materials, where $\boldsymbol{u}^{0}$ is the classical solution of (6) which, in addition, satisfies the following interfacial conditions:

$\left[\boldsymbol{u}^{0}\right]_{-}{ }^{+}=\left[\boldsymbol{a}^{0} \boldsymbol{n}\right]_{-}{ }^{+}=0 \quad$ at the interface.

Proof: As before, the vector field $\left(1-c \nabla^{2}\right) \boldsymbol{u}$ satisfies the classical problem (6), as well as the continuity condition (26.2) for the stress. Therefore, it remains to prove that the classical continuity condition (26.1) for the displacement is indeed satisfied by $\left(1-c \nabla^{2}\right) \boldsymbol{u}$.

To this end, we notice that for $c_{1}=c_{2}=c$ we have

$\left[\left(1-c \nabla^{2}\right) u\right]_{-}^{+}=[u]_{-}^{+}-c\left[\nabla^{2} u\right]_{-}^{+}=[u]_{-}^{+}-c\left[\frac{\partial^{2} u}{\partial x^{2}}+\frac{\partial^{2} u}{\partial z^{2}}\right]_{-}^{+}$,

1 The first two of (25) are the usual interface conditions for $c \equiv 0$, while (25.3) and (27) are the extra two conditions required for interfacial problems for the case $c \neq 0$. 
where the condition (25.3) has been used. Moreover, in view of the fact that

$\left[\frac{\partial^{2} \boldsymbol{u}}{\partial x^{2}}+\frac{\partial^{2} \boldsymbol{u}}{\partial z^{2}}\right]_{-}^{+}=\frac{\partial^{2}[\boldsymbol{u}]_{-}^{+}}{\partial x^{2}}+\frac{\partial^{2}[\boldsymbol{u}]_{-}^{+}}{\partial z^{2}}$

it follows from (25.1) that

$\left[\left(1-c \nabla^{2}\right) u\right]_{-}^{+}=0$

i.e., $\left(1-c \nabla^{2}\right) \boldsymbol{u}$ is continuous across the interface.

It must be emphasized that the conclusions of Theorem 3 have nothing to do with the second interfacial condition that is required for treating interfaces within the framework of gradient elasticity.

On the other hand, if we require

$\left[\frac{\partial \boldsymbol{u}}{\partial n}\right]_{-}^{+}=0 \quad$ at the interface

to be the additional interfacial condition, then it is not difficult to prove that Theorem 3 is still true for any curved interface. In fact, we can verify that the vector field $\left(1-c V^{2}\right) \boldsymbol{u}$, where $\boldsymbol{u}$ satisfies (25) and (27) at the interface, is continuous across any curved interface. It should be emphasized that condition $c_{1}=c_{2}=c$ is essential for the validity of Theorem 3 . Thus, under the conditions described in Theorem $3, \boldsymbol{u}$ may be obtained as the solution of the following boundary value problem:

$\left(1-c \nabla^{2}\right) \boldsymbol{u}=\boldsymbol{u}^{0} \quad$ in $\boldsymbol{V}$

$\frac{\partial^{2} u}{\partial n^{2}}=0 \quad$ on $S$

$\left[\frac{\partial^{2} \boldsymbol{u}}{\partial n^{2}}\right]_{-}^{+}=0, \quad\left[\frac{\partial u}{\partial n}\right]_{-}^{+}=0 \quad$ at the interface

This result is particularly useful in discussing the implications of gradient elasticity in the overlapping problem of interfacial crack surfaces. In fact, let us assume that an interface crack is located at $y=0,-a \leqq x \leqq a$, and that $c_{1}=c_{2}=c$. Then, from Theorem 3 and (28), we still have relationship (15), where $g(x)$ and $\boldsymbol{g}^{0}(x)$ are defined in Section 3.1. The expression of $\boldsymbol{g}^{\circ}(x)$ may be found in [7]; e.g. for Mode-I we have

$g_{y}{ }^{0}(x)=b \sqrt{a^{2}-x^{2}} \cos \gamma \theta$

where

$\theta=\frac{1}{2} \log \left(\frac{a+x}{a-x}\right)$

and the constants $b$ and $\gamma$ depend on the elastic constants of the two bonded bodies. It is easily seen that the first derivative of $g_{y}{ }^{0}$ changes sign and approaches infinity at the crack-tip. This means that the classical solution predicts interpenetration of the crack surfaces. 
On the other hand, from (19) and (29) we obtain the gradient crack-opening displacement as follows:

$$
\begin{aligned}
g_{y}(x)= & \frac{b \operatorname{sh}\left(\frac{a+x}{\sqrt{c}}\right)}{\sqrt{c} \operatorname{sh}\left(\frac{2 a}{\sqrt{c}}\right)} \int_{-a}^{a} \operatorname{sh}\left(\frac{a-t}{\sqrt{c}}\right) \sqrt{a^{2}-t^{2}} \cos \left[\frac{\gamma}{2} \log \left(\frac{a+t}{a-t}\right)\right] d t \\
& -\frac{b}{\sqrt{c}} \int_{-a}^{x} \operatorname{sh}\left(\frac{x-t}{\sqrt{c}}\right) \sqrt{a^{2}-t^{2}} \cos \left[\frac{\gamma}{2} \log \left(\frac{a+t}{a-t}\right)\right] d t .
\end{aligned}
$$

It is seen, in particular, that the first derivative of $g_{y}$ approaches a definite finite value, i.e.

$\boldsymbol{g}_{y}^{\prime}(-a)=\frac{b}{c \operatorname{sh}\left(\frac{2 a}{\sqrt{c}}\right)} \int_{-a}^{a} \operatorname{sh}\left(\frac{a-t}{\sqrt{c}}\right) \sqrt{a^{2}-t^{2}} \cos \left[\frac{\gamma}{2} \log \left(\frac{a+t}{a-t}\right)\right] d t$,

which obviously eliminates the interpenetration at the crack tip if $g_{y}{ }^{\prime}(-a)>0$.

To establish the positivity of the slope $g_{y}{ }^{\prime}(-a)$ of the crack profile at the tip, we note that

$$
\int_{-a}^{a}\left(e^{t / \sqrt{c}}-e^{(-t) / / \sqrt{c}}\right) \sqrt{a^{2}-t^{2}} \cos \left[\frac{\gamma}{2} \log \left(\frac{a+t}{a-t}\right)\right] d t=0
$$

and also recall $(b, c)>0$. With the above identity, it can be shown from $(31)$ that $g_{y}{ }^{\prime}(-a)$ has the same sign as the integral

$$
I \equiv \int_{-a}^{a} e^{t / V \bar{c}} \sqrt{a^{2}-t^{2}} \cos \left[\frac{\gamma}{2} \log \left(\frac{a+t}{a-t}\right)\right] d t .
$$
To determine the sign of $I$, we can define a point $x_{0} \equiv \frac{a\left(e^{M}-1\right)}{\left(e^{M}+1\right)}$ with $M \equiv \frac{2 \pi}{3|\gamma|}$ such that
$0<x_{0}<a$ and $\cos \left[\frac{y}{2} \log \left(\frac{a+x}{a-x}\right)\right]>\frac{1}{2}, \quad-x_{0} \leqq x \leqq x_{0}$.

It follows that

$$
\begin{aligned}
& I>\frac{1}{2} \int_{0}^{x_{0}} e^{t / V \bar{c}} \sqrt{a^{2}-t^{2}} d t-\int_{-a}^{-x_{0}} e^{t / / \bar{c}} \sqrt{a^{2}-t^{2}} d t-\int_{x_{0}}^{a} e^{t / / \bar{c}} \sqrt{a^{2}-t^{2}} d t \\
& >2 \sqrt{a^{2}-x_{0}^{2}}\left[\frac{\sqrt{c}\left(e^{x_{0} / V \bar{c}}-1\right)}{4}-\left(a-x_{0}\right) e^{a / V \bar{c}}\right] .
\end{aligned}
$$

It thus turns out that the following sufficient condition is derived to guarantee the positivity of $I$ : $\frac{\sqrt{c}}{a}>8 e^{-M}, \quad$ for $e^{a / \sqrt{c}} \gg 1 \quad$ and $e^{M} \gg 1$, 
suggesting that the gradient term removes the "interpenetration" problem near the interfacial crack tip. Similar conclusions may be drawn for a Mode-II crack with $g_{y}{ }^{0}=b \sqrt{a^{2}-x^{2}} \sin \gamma \theta$. More details will be included in a forthcoming paper [8].

\section{Acknowledgements}

The financial support of NSF under grant MSS-890700 and AFOSR under grant AFOSR-91-0421 is gratefully acknowledged.

\section{References}

[1] Aifantis, E. C.: On the microstructural origin of certain inelastic models. Trans. ASME. J. Mat. Eng. Tech. 106, 326-330 (1984).

[2] Aifantis, E. C.: The physics of plastic deformation. Int. J. Plasticity 3, 211-247 (1987).

[3] Triantafyllidis, N., Aifantis, E. C.: A gradient approach to localization of deformation-I. Hyperelastic materials. J. Elasticity 16, 225-238 (1986).

[4] Zbib, H., Aifantis, E. C.: On the localization and post-localization of plastic deformation. Part I. On the initiation of shear-bands; Part II. On the evolution and thickness of shear bands; Part III. On the structure and velocity of Portevin-Le Chatelier bands. Res Mechanica 23 (Special Issue on Material Instabilities, Aifantis, E. C. et al., eds.), 261 - 277, 279-292, 293-305 (1988).

[5] Altan, S. B., Aifantis, E. C.: On the structure of the mode-III crack-tip in gradient elasticity. Scripta Met. 26, $319-324$ (1992).

[6] Love, A. E. H.: A treatise on mathematical theory of elasticity. New York: Dover 1944.

[7] Sneddon, I. N.: Application of integral transforms in the theory of elasticity. CISM, No. 220, 1975.

[8] Ru, C. Q., Aifantis, E. C.: In preparation.

Authors' address: C. Q. Ru* and E. C. Aifantis**, Department of Mechanical Engineering and Engineering Mechanics, Michigan Technological University, Houghton, MI 49931, U.S.A.

* Permanent address: Institute of Mechanics, Chinese Academy of Sciences, Beijing 100080, People's Republic of China

** Also: Aristotle University of Thessaloniki, Thessaloniki, GR 54006, Greece 